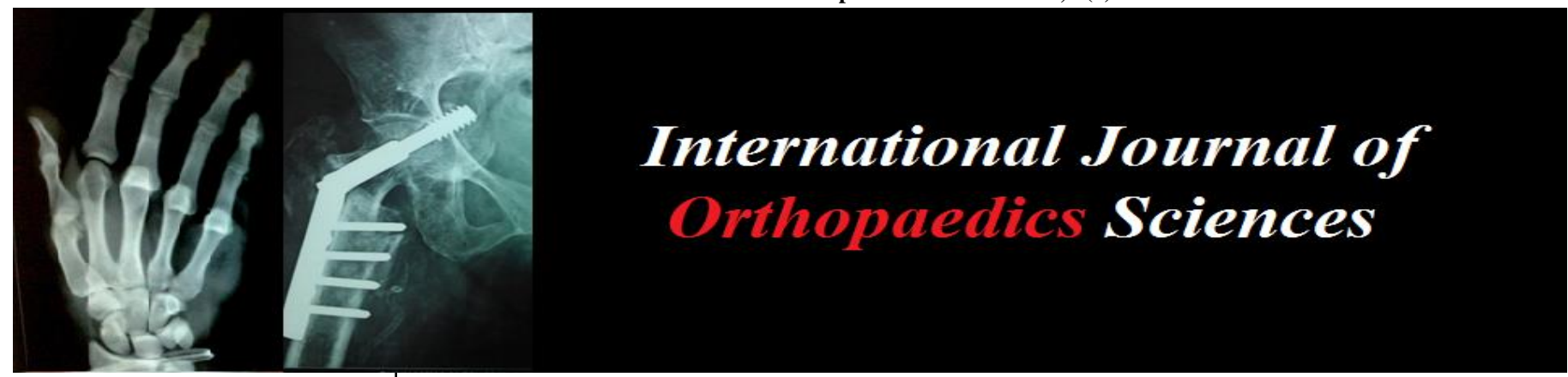

ISSN: $2395-1958$

IJOS 2018; 4(3): 423-425

(C) 2018 IJOS

www.orthopaper.com

Received: 16-05-2018

Accepted: 17-06-2018

Dr. Ashwini Sankhe

Lokmanya Tilak Municipal

Medical College \& General

Hospital, Sion, Mumbai,

Maharashtra, India

Dr. Sukhada Kulkarni

Lokmanya Tilak Municipal

Medical College \& General

Hospital, Sion, Mumbai,

Maharashtra, India

Dr. Arvind Borde

Lokmanya Tilak Municipal

Medical College \& General

Hospital, Sion, Mumbai,

Maharashtra, India

Dr. Salman Mapara

Lokmanya Tilak Municipal

Medical College \& General

Hospital, Sion, Mumbai,

Maharashtra, India
Correspondence

Dr. Sukhada Kulkarni

Lokmanya Tilak Municipal

Medical College \& General

Hospital, Sion, Mumbai,

Maharashtra, India

\section{Calculation and evaluation of angle of acetabular anteversion in normal healthy Indian population}

\author{
Dr. Ashwini Sankhe, Dr. Sukhada Kulkarni, Dr. Arvind Borde and \\ Dr. Salman Mapara
}

DOI: https://doi.org/10.22271/ortho.2018.v4.i3h.75

\section{Abstract}

Studies have been carried out regarding the acetabular and femoral anteversion in patients with developmental dysplasia of hip. However, definitive criteria/measurements regarding the normal angle of acetabular anteversion in asymptomatic patients is lacking. In our study, angle of anteversion of the acetabulum was determined by use of computed tomography in 200 asymptomatic Indian adults. The overall normal anteversion of the acetabulum was found to be $16.43 \pm 6{ }^{0}$ (mean \pm standard deviation) with no significant sex related difference. Slight age related increase in the angle of acetabular anteversion was observed.

Keywords: CT, Hip, acetabulum, femur, anteversion

\section{Introduction}

The hip joint has major role in maintenance of the erect posture of human beings. The relationship of the acetabulum and the femoral neck and head influences the biomechanics of the hip joint. Anteversion of both the femur and the acetabulum is valuable in the evaluation of normal as well as pathological conditions of the hip ${ }^{[1]}$.

Recognition, understanding and treatment of pathologic conditions in the hip joint must be supported by exact knowledge of the normal values of these parameters, both in growing children, as well as in skeletally mature patients ${ }^{[2,3,4]}$.

The mechanics of the hip joint is dependent on the relationship between the femoral head and the acetabulum [1, 2, 5]. Abnormal femoral neck anteversion (FNA) and/or acetabulum anteversion (AA) have been implicated in the etiogenesis of osteoarthritis, developmental dysplasia of the hip (DDH), and impingement, instability and wear in total hip arthroplasty (THA) ${ }^{[1,2,5]}$.

A review of the global literature reveals a wide range of normal FNA and AA with racial and geographic variation ${ }^{[2,5]}$. This variation is expected to exist because of different social needs of the different races. Numerous studies have focused on FNA in the normal population; however, little attention has been given to the normal AA. Moreover, studies on the Indian population are sparse on this topic ${ }^{[5-8]}$. Since Indians are more prone to indulge in floor level activities like squatting and sitting cross-legged, the hip is flexed, externally rotated and abducted to the extremes of motion. We were interested whether this resulted in morphologically different hip anteversion in Indians as compared to the Western population. The purpose of this study was to define a normal range of values for AA.

\section{Material and Method}

We have included patients who came to our department for CT scan of abdomen, pelvis or other scans acquired exclusively for medical indications. All patients were evaluated using a Philips Brilliance 64 slice CT scanner (Mumbai, India). A total of (200) thin-slice CT datasets of right or left femurs of skeletally mature patients were analyzed in a cross-sectional study. 


\section{Aims and Objectives}

1. To calculate the angle of femoral anteversion in normal asymptomatic population having no hip related complaints

2. To identify any demographic variations in the normal angle of anteversion like the effect of age and gender.

\section{Inclusion criteria}

Adults who had a computed tomographic (CT) scan of the pelvis for pathology unrelated to the hip. Patients with complete bony fusion of the acetabulum were only included.

\section{Exclusion criteria}

1. Young patients/patients with incomplete fusion of the acetabulum,

2. Patients with bony pathology of the pelvis and femur including fractures or deformities,

3. Patients with hip pathology as evident clinically with gait abnormality and/or pain/restriction of hip motions,

4. Prior surgical intervention and patients with hardware in situ,

5. Childhood hip, knee or spine disease

6. Patients with current or previous metabolic bone disease, and

7. Uncooperative patients.
We defined angle of acetabular version as the angle between a line connecting the lateral anterior and posterior margins of the acetabular component and the sagittal plane defined as the plane perpendicular to a line connecting two identical points on either side of the pelvis ${ }^{[9]}$.

\section{Image analysis}

Initially the scans were evaluated in soft tissue and bone window settings for assessing any signs of hip joint pathology or other pathologies which are likely to result in hip joint orientation and hence change of acetabular anteversion angle. Patients who revealed hip joint effusion, osteoarthritic changes or psoas abscess etc. were rejected. Followed by this, the scans were aligned with respect to the lumbar spinal alignment in sagittal and coronal sections. Then sequential axial sections were analyzed in bone window settings and the best sections revealing the section of pelvis with round femoral head, anterior and lateral margins of acetabulum was selected for measurement of acetabular anteversion angle. A plane sagittal to the pelvis was taken and any line connecting two identical points on either sides was considered as the horizontal reference line. We took identical points at the level of acetabulum.

We used the same definition as Maheshwari AV et al. (5) for measurement of the acetabular anteversion angle. A prototype measurement is depicted below.

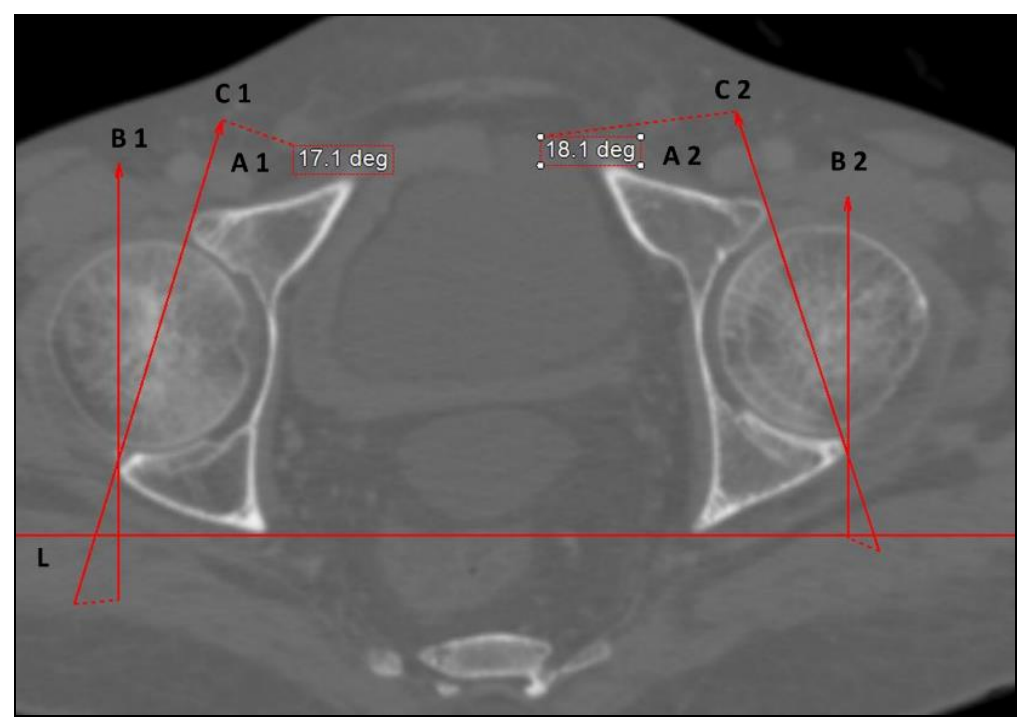

Line L: Horizontal line connecting two identical points on either side of the pelvis, marking the sagittal plane

$\mathrm{B}$ : Line perpendicular to line $\mathrm{L}$

$\mathrm{C}$ : line connecting the lateral anterior and posterior margins of the acetabular component

A: Angle of acetabular anteversion, calculated between lines $\mathrm{B}$ and $\mathrm{C}$

Precise angle of acetabular anteversion were obtained in patients. Measurements due to human error were minimized by simultaneous measurement by two analysts. There were 94 male and 106 female patients. The mean angle of acetabular anteversion was $16.43 \pm 6^{\circ}$. The mean age of patients was 52 years.

\section{Results}

Of these 200 patients, there were 94 male and 106 female patients (Distribution depicted in Pie chart). The average age was 52 years with the maximum age being 82 years and minimum being 22 years.

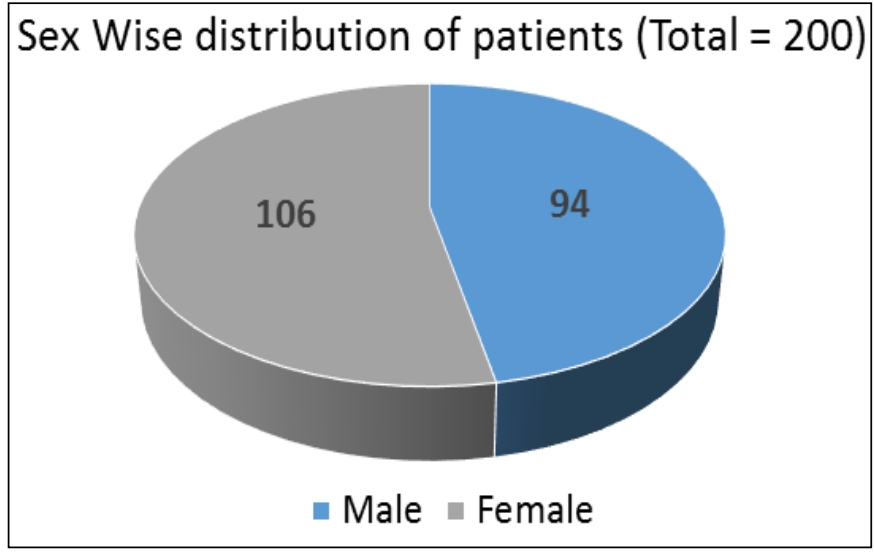

Precise angle of acetabular anteversion was obtained in patients. The overall mean angle of acetabular anteversion was $16.43^{\circ}$. For females, the mean angle was $16.92^{\circ}$ and for males it was $15.94^{\circ}$. Hence there is no significant gender related differences at $95 \%$ confidence interval. 
Age related mean angles in female and male patients are given in below mentioned tables.

Age wise distribution of mean angle of acetabular anteversion in male patients is given in below mentioned table. The average angle of acetabular anteversion in male patients on right side was $18.1^{\circ}$ and on left side was $16.2^{\circ}$.

\begin{tabular}{|c|c|c|}
\hline Age & Right & Left \\
\hline $21-30(1)$ & 16.68 & 17.31 \\
\hline $31-40(2)$ & 18.5 & 16.5 \\
\hline $41-50(3)$ & 16.85 & 14.4 \\
\hline $51-60(4)$ & 16.87 & 14.59 \\
\hline $61-70(5)$ & 16.61 & 14.76 \\
\hline $71-80(6)$ & 17.2 & 13.9 \\
\hline $81-90(7)$ & 24.5 & 22 \\
\hline \multicolumn{2}{|l}{} \\
\hline
\end{tabular}

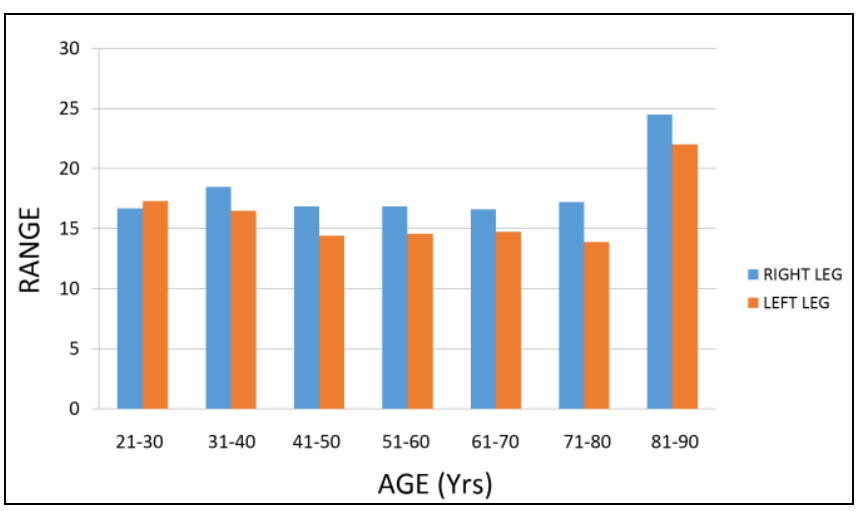

Age wise distribution of mean angle of acetabular anteversion in female patients is given in below mentioned table. The average angle of acetabular anteversion in female patients on right side was $18.8^{\circ}$ and on left side was $17.7^{\circ}$.

\begin{tabular}{|c|c|c|}
\hline Age & Right & Left \\
\hline $21-30(1)$ & 16.72 & 13.63 \\
\hline $31-40(2)$ & 19.63 & 16.8 \\
\hline $41-50(3)$ & 19.55 & 16.47 \\
\hline $51-60(4)$ & 15.33 & 16.41 \\
\hline $61-70(5)$ & 16.83 & 16.41 \\
\hline $71-80(6)$ & 20.66 & 22 \\
\hline $81-90(7)$ & 23 & 22 \\
\hline
\end{tabular}

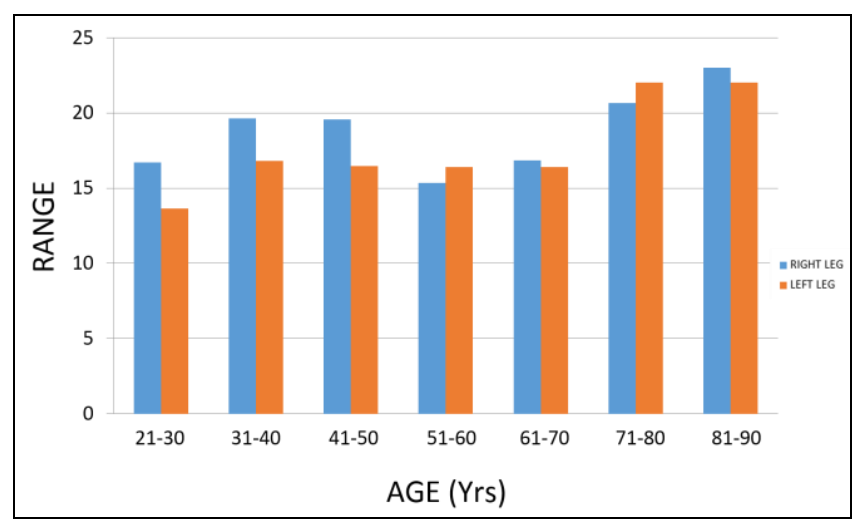

The acetabular angle of anteversion was observed to be more in the age group of 81-90 years in both, males as well as females. This can be attributed to the bone loss. However, the sample size in this age group was less i.e. 2 male patients and 1 female patient, hence may not be conclusive and needs further study.

\section{Conclusion}

Studies have been carried out regarding the acetabular and femoral anteversion in patients with hip related pathologies. However, definitive criteria/measurements regarding the normal angle of acetabular anteversion in asymptomatic patients is lacking. More so, there is no large scale study of Indian population regarding the same. The purpose of this study was to determine the acetabular anteversion in asymptomatic Indian adult population.

In our study, anteversion of the acetabulum was determined by use of computed tomography in 200 asymptomatic Indian adults. The normal anteversion of the acetabulum was found to be $16.43 \pm 6^{0}$ (mean \pm standard deviation) with the angle being $1^{0}$ less in females as compared to males, however this is not statistically significant. The acetabular angle of anteversion was observed to be more in the age group of 8190 years, which can be attributed to the bone loss. However, the sample size in this age group was less hence may not be conclusive and hence needs further study.

\section{References}

1. Reikeråls O, Bjerkreim I, Kolbenstvedt A. Anteversion of the acetabulum and femoral neck in normals and in patients with osteoarthritis of the hip. Acta Orthopaedica Scandinavica. 1983; 54(1):18-23.

2. Tönnis D, Heinecke A. Current Concepts ReviewAcetabular and Femoral Anteversion: Relationship with Osteoarthritis of the Hip. J Bone Jt Surg. 1999; 81:174770.

3. Ejnisman L, Philippon MJ, Lertwanich P, Pennock AT, Herzog MM, Briggs KK et al. Relationship Between Femoral Anteversion and Findings in Hips With Femoroacetabular Impingement. Orthopedics. 2013; 36:e293-e300.

4. Hartel MJ, Petersik A, Schmidt A, Kendoff D, Nüchtern $\mathrm{J}$, Rueger JM et al. Determination of femoral neck angle and torsion angle utilizing a novel three-dimensional modeling and analytical technology based on CT datasets. PloS one. 2016; 11(3):e0149480.

5. Maheshwari AV, Zlowodzki MP, Siram G, Jain AK. Femoral neck anteversion, acetabular anteversion and combined anteversion in the normal Indian adult population: A computed tomographic study. Indian Journal of Orthopaedics. 2010; 44(3):277.

6. Saikia K, Bhuyan S, Rongphar R. Anthropometric study of the hip joint in Northeastern region population with computed tomography scan. Indian J Orthop. 2008; 42:260-6.

7. Nagar M, Bhardwaj R, Prakash R. Anteversion in adult Indian femora. J Anat Soc India. 2002; 49:9-12.

8. Siwach RC, Dahiya S. Anthropometric study of proximal femur geometry and its clinical application. Indian J Orthop. 2003; 37:247-51.

9. Fujishiro T, Hayashi S, Kanzaki N, Hashimoto S, Kurosaka M, Kanno $\mathrm{T}$ et al. Computed tomographic measurement of acetabular and femoral component version in total hip arthroplasty. International Orthopaedics. 2014; 38(5):941-6. 